\title{
Thermionic-enhanced near-field thermophotovoltaics for medium-grade heat sources
}

\author{
A. Datas and R. Vaillon
}

\begin{abstract}
Conversion of medium-grade heat (temperature from 500 to $1000 \mathrm{~K}$ ) into electricity is important in applications such as waste heat recovery or power generation in solar thermal and co-generation systems. At such temperatures, current solid-state devices lack either high conversion efficiency (thermoelectrics) or high-power density capacity (thermophotovoltaics and thermionics). Near-field thermophotovoltaics (nTPV) theoretically enables high-power density and conversion efficiency by exploiting the enhancement of thermal radiation between a hot emitter and a photovoltaic cell separated by nanometric vacuum gaps. However, significant improvements are possible only at very small gap distances $(<100 \mathrm{~nm})$ and when ohmic losses in the photovoltaic cell are negligible. Both requirements are very challenging for current device designs. In this work, we present a thermionic-enhanced near-field thermophotovoltaic (nTiPV) converter consisting of a thermionic emitter (graphite) and a narrow bandgap photovoltaic cell (InAs) coated with low-workfunction nanodiamond films. Thermionic emission through the vacuum gap electrically interconnects the emitter with the front side of the photovoltaic cell and generates an additional thermionic voltage. This avoids the use of metal grids at the front of the cell and virtually eliminates the ohmic losses, which are unavoidable in realistic nTPV devices. We show that nTiPV operating at $1000 \mathrm{~K}$ and with a realizable vacuum gap distance of $100 \mathrm{~nm}$ enables a 10.7 -fold enhancement of electrical power $\left(6.73 \mathrm{~W} / \mathrm{cm}^{2}\right)$ and a 2.8 -fold enhancement of conversion efficiency (18\%) in comparison with a realistic nTPV device having a series resistance of $10 \mathrm{~m} \Omega \cdot \mathrm{cm}^{2}$.
\end{abstract}

Thermionics (TIC) $)^{1,2}$ and thermophotovoltaics (TPV) $)^{3,4}$ are highly efficient alternatives to thermoelectric generators (TEGs). ${ }^{5}$ In TIC, electrons are thermally emitted from a hot emitter/cathode and collected in a cold anode/collector, subsequently producing an electrical current. In TPV, thermally radiated photons are absorbed in a lowbandgap semiconductor and excite electron-hole pairs, which are selectively collected to produce an electrical current. Both TPV and TIC have already demonstrated higher conversion efficiencies than TEG at temperatures beyond $1000^{\circ} \mathrm{C}\left(\sim 24 \%\right.$ for $\mathrm{TPV}^{6,7}$ and $\sim 11 \%$ for $\mathrm{TIC}^{1}$ ). However, the power density is comparatively very low (e.g., less than $1 \mathrm{~W} / \mathrm{cm}^{2}$ for TPV at $1039^{\circ} \mathrm{C}^{6}$, while $\sim 20 \mathrm{~W} / \mathrm{cm}^{2}$ for TEG at $595^{\circ} \mathrm{C}^{8}$ ). The main reason is the lower energy flux of radiated particles compared to that carried by the electrons moved by a temperature gradient within a solid, as in TEG.

Boosting the power density of TIC and TPV is the motivation of current research efforts that aim at increasing the flux of radiated photons (for TPV) and electrons (for TIC). For TIC, most of the research focuses on reducing the workfunction of the emitter and the collector, along with the reduction in the accumulated space-charge by applying magnetic fields or by reducing the vacuum gap that separates the cathode and the anode to micrometric scales. ${ }^{1,2}$ For TPV, at least three strategies were proposed for increasing the energy flux of radiated photons at moderate temperatures: light-pipe TPV (LTPV), ${ }^{9}$ thermophotonics (TPX), ${ }^{10}$ and near-field thermophotovoltaics (nTPV). ${ }^{11}$ Recently, a combination of the last two has also been proposed. ${ }^{12}$ nTPV is the strategy with the highest theoretical potential. It consists of creating nanoscale vacuum gaps between the emitter and the photovoltaic (PV) cell, so that evanescent waves (photons) tunnel from the emitter to the cell and contribute to generating electrical power. Near-field thermal radiation transport was thoroughly investigated from both theoretical and experimental points of view, ${ }^{13,14}$ and its potential use for heat-to-electricity conversion was widely 
analyzed. ${ }^{15}$ Only, very recently, the proof-of-concept of nTPV has been finally achieved by measuring a 40 -fold enhancement of the electrical output power at gap distances of less than $100 \mathrm{~nm} .{ }^{16}$ However, nTPV has (at least) two main relevant issues that may impede its further deployment: first, the quite high ohmic losses due to the very high current densities that must flow laterally through thin semiconductor layers within the PV cell; second, the very small vacuum gaps that are needed to obtain a significant improvement in electrical power density.

In this work, we present a theoretical analysis of a thermionicenhanced nTPV device (nTiPV) that eliminates the ohmic losses and enables higher power densities at larger gap distances than conventional nTPV. It is the aim of this work to illustrate the theoretical potential of a specific device with a medium-grade heat source operating at a temperature of $1000 \mathrm{~K}$.

Figure 1 shows the band diagram of the proposed device, which is the near-field counterpart of the hybrid thermionic-photovoltaic concept introduced previously. ${ }^{17}$ The system consists of a graphite emitter and an InAs (with a bandgap of $0.35 \mathrm{eV}$ at $300 \mathrm{~K}$ ) PV cell separated by a distance $d$. The emitter and the PV cell are coated with very thin $(\sim 1-2 \mathrm{~nm})$ transparent H-terminated diamond films, which have been proved experimentally to provide workfunctions in the range of around $1.4 \mathrm{eV}$ ( $\mathrm{N}$-doped films ${ }^{18}$ ) down to around $0.9 \mathrm{eV}$ (P-doped films ${ }^{19}$ ). The emitter is heated by an external heat source and subsequently radiates photons and electrons towards the PV cell. Due to the emission of electrons, the emitter surface is charged positively. Thus, the radiated electrons are attracted back, and if the distance $d$ is relatively large, they accumulate in the vacuum gap. This regime of operation is named the "space-charge-limited" mode, and it is characterized by

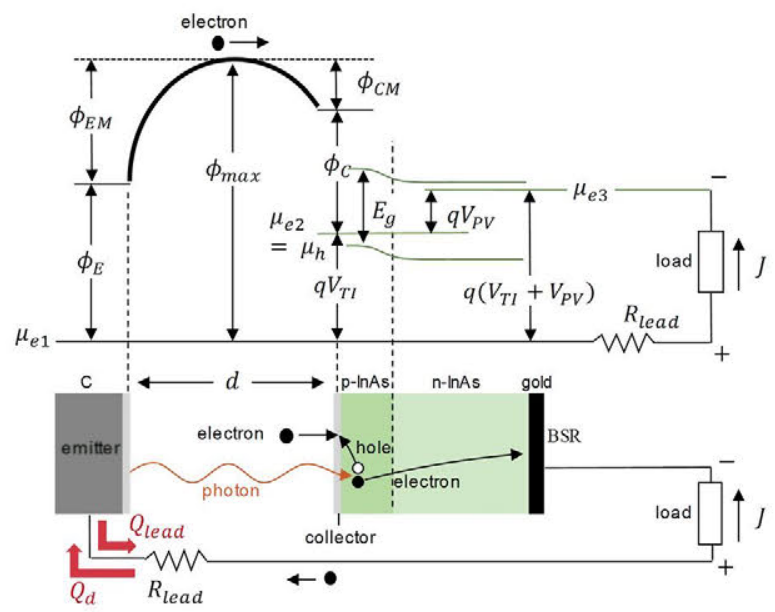

FIG. 1. Band diagram of the proposed nTiPV device comprising a $C$ thermal emitter and an InAs PV cell with engineered low-workfunction materials and/or coatings. The thermally excited electrons having enough energy to overcome the emitter workfunction $\phi_{E}$ and the space-charge barrier $\phi_{E M}$ are radiated towards the InAs PV cell, which is separated by distance $d$ from the emitter. The electrons are collected at the PV cell surface, also named the collector, which is biased at voltage $V_{T I}$. The photons are absorbed within the PV cell and generate an electron-hole pair. The photogenerated holes recombine with the thermionically collected electrons coming from the emitter. The photogenerated electrons are collected in the rear contact, which also comprises a gold back surface reflector (BSR). The electrochemical potential of electrons gradually increases from $\mu_{\theta 1}$ (when injected into the emitter from the lead) to $\mu_{\mathrm{e} 2}$ (after being collected in the PV cell surface) and finally to $\mu_{\mathrm{e} 3}$ (when collected in the rear terminal of the PV cell). additional potential barriers $\phi_{\mathrm{EM}}$ and $\phi_{\mathrm{CM}}$ that oppose the electrons' flow. In contrast, if the distance $d$ is small, the radiated electrons are effectively collected at the InAs PV cell surface, without accumulating in the gap, subsequently eliminating any kind of potential barrier and leading to a drastic enhancement of the thermionic current. When the thermionically emitted electrons reach the PV cell surface, they recombine with the holes photogenerated in the PV cell. Ideally, no electrical potential is created in this process, as in ideal ohmic contacts. Therefore, the full PV cell front side behaves as a transparent collector that ensures the wireless electrical connection between the emitter and the PV cell. The output voltage is thus the addition of the thermionic voltage $\left(V_{T I}\right.$, generated between the emitter and the front side of the PV cell) and the photovoltaic voltage $\left(V_{P V}\right.$, generated between the front and rear sides of the PV cell). Remarkably, this design avoids the use of front metal grids, eliminating the subsequent shadowing losses and mitigating the challenges of nano-gap implementation in space-constrained near-field TPV devices. In conventional PV cells, either in front- or backcontacted configurations, the main contributors to the ohmic losses are the currents that flow laterally through the semiconductor and metal layers. These losses are fully eliminated in the nTiPV device, where the current flow is nearly unidirectional and transversal to the device's area. The results will show that this is a particularly significant benefit for near-field operation, which involves very large current densities.

The far-field counterpart of this device ${ }^{17}$ is being experimentally developed. $^{20,21}$ The experimental device operates under ultra-high vacuum (UHV) conditions and uses dielectric micro-spacers to create a micrometer vacuum gap between the emitter and the PV cell. Modern microfabrication techniques already enabled the development of thermally and electrically insulated spacers that withstand large temperature gradients. These techniques eventually enabled the experimental demonstration of micron-gap TIC. ${ }^{22,23}$ Sub-micron separation distances were also experimentally realized using nano-spacers in the frame of near-field thermal radiation experiments. ${ }^{24-27}$ Despite the recent experimental demonstration of $n \mathrm{nPV}^{16}$ realized using a suspended emitter and precise alignment tools, current research efforts target the integration of spacers into stable nano-gap nTPV devices. ${ }^{28}$ All the recent progress should be directly transferrable to the experimental implementation of nTiPV devices.

Analysis of the nTiPV device described above requires the calculation of the total net flux of photons and electrons through nanoscale vacuum gaps, along with the generated current-voltage characteristics of both thermionic and photovoltaic converters in the near-field.

For the thermionic part, the electrons' energy flux $\left(Q_{e l}\right)$, the generated current density $\left(J_{T I}\right)$, and the output voltage $\left(V_{T I}\right)$ can be calculated by neglecting collector's back emission as ${ }^{29}$

$$
\begin{gathered}
Q_{e l}=J_{T I} \frac{\left(\phi_{\max }+2 k T_{E}\right)}{e}, \\
J_{T I}=A T_{E}^{2} e^{\frac{-q \phi_{\max }}{k T_{E}}}, \\
q V_{T I}=\phi_{E}+\phi_{E M}-\phi_{C}-\phi_{C M},
\end{gathered}
$$

where $A$ is the Richardson-Dushman constant, $k$ is the Boltzmann constant, $q$ is the electron's charge, and $T_{E}$ is the emitter temperature. $\phi_{\max }$ is the maximum of the electric potential created along the interelectrode gap (Fig. 1). In the space-charge-limited mode, $\phi_{\max }=\phi_{E}$ $+\phi_{E M}$ or $\phi_{\max }=q V_{T I}+\phi_{C}+\phi_{C M}$, with $\phi_{E}\left(\phi_{C}\right)$ being the emitter's (collector's) workfunction. The values of energy barriers $\phi_{E M}$ and $\phi_{C M}$ 
can be calculated using Langmuir theory. ${ }^{29}$ This theory assumes onedimensional and collision-less electron flow with a half-Maxwellian distribution of velocities. In the so-called retarding mode, $V_{T I}$ is large enough to locate the maximum of the electrostatic potential at the collector's surface, i.e., $\phi_{\max }=q V_{T I}+\phi_{C}$. The latter will be the most typical case in the near-field, where the very small inter-electrode distance will nearly eliminate the barriers $\phi_{E M}$ and $\phi_{C M}$ and the maximum power point (MPP) will occur at $\phi_{\max } \approx \phi_{E}$ and $q V_{T I} \approx \phi_{E}$ $-\phi_{C}$. In order to analyze the theoretical potential of the concept, a Richardson constant of $120 \mathrm{~A} / \mathrm{cm}^{2}$ is assumed. Significant deviations from this theoretical value are possible depending on the experimental conditions of the deposition of the emitter film, as well as on the interfacial layers that could be created during this process. ${ }^{18}$

For the photovoltaic part, the photons' energy flux is calculated using fluctuational electrodynamics ${ }^{30}$ and the S-matrix method for 1Dlayered media. ${ }^{31}$ The current density $\left(J_{P V}\right)$-voltage $\left(V_{P V}\right)$ characteristic is calculated by solving the minority carrier diffusion equation in the frame of the low-injection approximation, using the methods described elsewhere. ${ }^{32,33}$ The device consists of four layers sandwiched between two semi-infinite media, respectively, made of graphite (emitter, semiinfinite), vacuum (gap with variable thickness $d$ ), p-doped InAs $\left(N_{a}=10^{18} \mathrm{~cm}^{-3}, 0.75 \mu\right.$ m thick), n-doped InAs $\left(N_{d}=10^{16} \mathrm{~cm}^{-3}, 6 \mu \mathrm{m}\right.$ thick), gold [back surface reflector (BSR), $200 \mathrm{~nm}$ thick], and vacuum (semi-infinite). Radiative, Auger, and Shockley-Read-Hall (SRH) recombination mechanisms are considered with parameters from Ref. 34, along with finite doping and temperature-dependent mobilities for electrons and holes. ${ }^{35}$ The model assumes that the thermionic layer on the PV cell does not modify the PV cell band diagram in a way that holes could not diffuse towards the thermionic collector. This is a reasonable assumption given the presence of electrically active defects at the semiconductor-diamond interface, as well as the very high doping levels of both the p-doped InAs layer and the diamond thin film, both effects preventing the creation of Schottky barriers at the semiconductordiamond interface. ${ }^{18,36,37}$ The optical properties of InAs corresponding to interband absorption and interactions with free carriers and phonons are calculated using the method described in Ref. 38 and the DrudeLorentz model, ${ }^{39}$ respectively, with the parameters of Ref. 40 . The Drude model is used for gold. ${ }^{41}$ For the sake of simplicity, diamond layers are omitted in the radiation transfer calculations. Their impact on emission by the graphite emitter and absorption by the InAs cell is assumed to be negligible, because the layers are very thin $(\sim 1-2 \mathrm{~nm})$, diamond's extinction coefficient is weak, and diamond's refractive index is of intermediate level between that of graphite and indium arsenide. ${ }^{42}$

Finally, the nTiPV conversion efficiency is given by

$$
\eta=\frac{\left[S J\left(V_{T I}+V_{P V}-S J R_{\text {lead }}\right)\right]_{\max }}{S\left(Q_{e l}+Q_{p h}\right)+Q_{\text {lead }}-Q_{d}},
$$

where $\left[S J\left(V_{T I}+V_{P V}-S J R_{\text {lead }}\right)\right]_{\max }$ is the maximum electrical power at the current density $J=J_{T T}=J_{P V}$ and the voltage $V=V_{T I}+V_{P V}$ $-S J R_{\text {lead }} . Q_{\text {lead }}=L\left(T_{E}^{2}-T_{C}^{2}\right) / 2 R_{\text {lead }}$ is the minimum amount of heat lost through the emitter's leads having an electrical resistance $R_{\text {lead }}$, $L=\frac{\pi^{2} k^{2}}{3 e^{2}}$ is the Lorentz number of the metal, ${ }^{29}$ and $Q_{d}=\mathrm{S}^{2} J^{2} R_{\text {lead }} / 2$ represents the heat generated in the leads by the Joule effect that is turned back to the emitter, with $S$ being the device area, equal to $1 \mathrm{~cm}^{2}$ in the current study. The value of $R_{\text {lead }}$ can be optimized to fulfill a trade-off between heat losses and power generation that ultimately maximizes the conversion efficiency. For comparison purposes, the conversion efficiency of a standalone nTPV device is calculated as $\eta_{n T P V}=\left[J\left(V_{P V}-J_{P V} R_{s}\right)\right]_{\max } / Q_{p h}$, with $R_{s}$ being the PV cell series resistance in $\Omega \cdot \mathrm{cm}^{2}$. In every calculation involving the search for maximum values, the Nelder-Mead algorithm is used. ${ }^{4}$

Figures 2(a) and 2(b) show the generated current density (voltage) of the nTiPV device as a function of gap distance $d$. The results are shown for two values of the emitter workfunction $\left(\phi_{E}=1.3\right.$ and $1.4 \mathrm{eV})$, a fixed collector's workfunction $\left(\phi_{C}=1 \mathrm{eV}\right)$, and an emitter temperature of $T_{E}=1000 \mathrm{~K}$. The lead resistance $\left(R_{\text {lead }}\right)$ is optimized at every distance to maximize the n'TiPV conversion efficiency. The voltage generated in the photovoltaic $\left(V_{P V}\right)$ and thermionic $\left(V_{T I}\right)$ stages is also shown in Fig. 2(b), along with the voltage drop in the leads $\left(-J \cdot R_{\text {lead }}\right)$. As explained earlier, both thermionic and photovoltaic currents must be identical within the nTiPV device due to the series interconnection, i.e., $J=J_{T I}=J_{P V}$. This means that their respective internal voltages, $V_{T I}$ and $V_{P V}$ [see Fig. 2(b)], must be adapted to meet this condition. However, the maximum power density
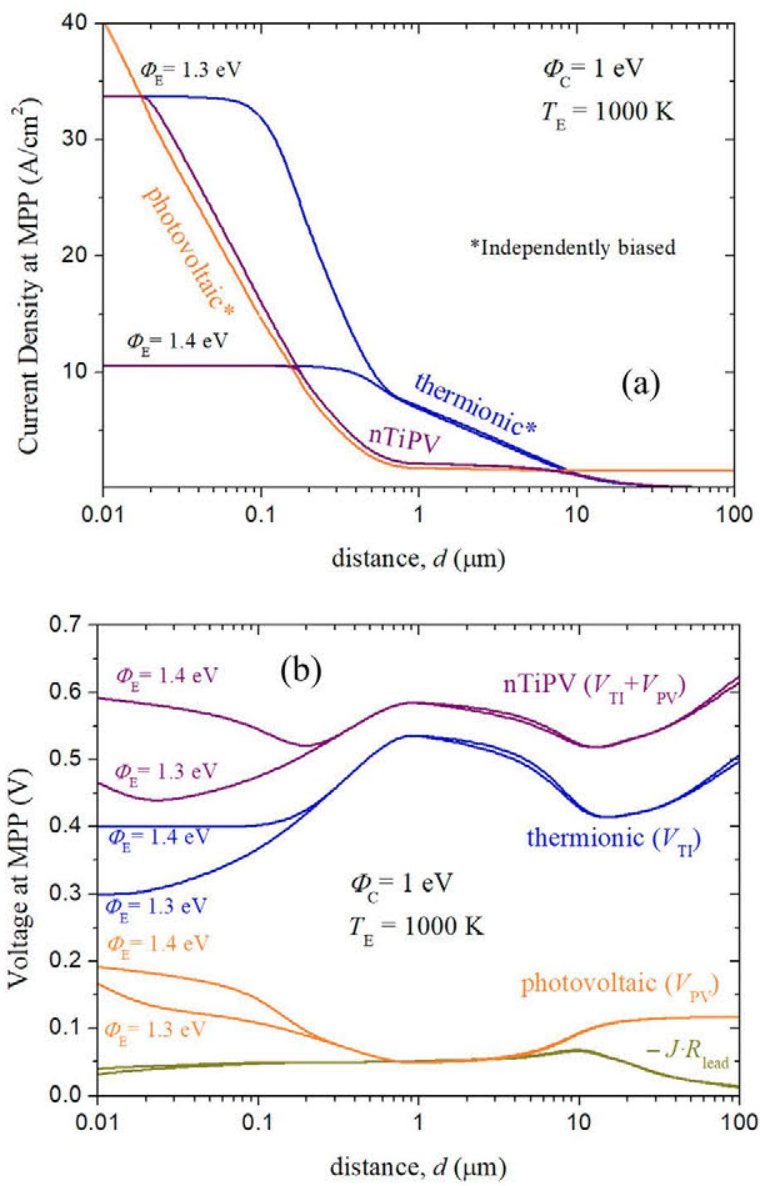

FIG. 2. Current densities (a) and voltages (b) of nTiPV as a function of gap distance between the emitter and the PV cell. The current densities of the photovoltaic and thermionic sub-devices are identical in the nTiPV device, but independently biased photovoltaic and thermionic current densities are shown in (a) to illustrate which of them is limiting the total device current. Different workfunctions of the emitter $\left(\phi_{\mathrm{E}}\right)$ are considered. $R_{\text {lead }}$ is optimized at every distance to maximize the nTiPV conversion efficiency. The device area is $1 \mathrm{~cm}^{2}$. 
attainable for each sub-device would be higher if they were biased independently. This is illustrated in Fig. 2(a), which also shows the current densities at the MPP for the independently biased thermionic $\left(J_{T I}^{*}\right)$ and photovoltaic $\left(J_{P V}^{*}\right)$ devices. This information is valuable for the following discussion.

There are three main different regions in Fig. 2(a). For large distances, thermionic emission is strongly reduced by the space-charge effect, also illustrated by the larger thermionic voltage contribution in Fig. 2(b), and limits the total current of the nTiPV device. This causes the PV cell to be biased near the open circuit. For intermediate distances, the space charge is mitigated and the flux of thermionically emitted electrons exceeds that of photogenerated charges in the PV cell. This causes an increase (decrease) in the thermionic (photovoltaic) voltage that reduces (increases) the thermionic (photovoltaic) current until both thermionic and photovoltaic currents are identical. In this region, the thermionic device undergoes the transition from the spacecharge-limited to saturation mode, and the photovoltaic device undergoes the transition from the far field to the near-field. The third region corresponds to the smallest distances at which photovoltaic photogeneration exceeds the thermionic electrons' flux due to the strong nearfield enhancement of photons' flux. In this region, the thermionic current is already saturated, with no space-charge effect, and limits the total current of the nTiPV device. Saturation of the thermionic subdevice is characterized by voltages approaching $V_{T I}=\phi_{E}-\phi_{C}$ at very small distances $[0.3$ and $0.4 \mathrm{eV}$ in Fig. 2(b)]. The transitions through these three regimes take place at two specific distances for which both thermionic and photovoltaic currents are equal. There is one in the near-field and another in the far field. Only at these specific distances, both thermionic and photovoltaic subdevices are biased simultaneously at their respective MPP. In all other situations, one of the devices produces a lower current, and it is consequently biased at larger voltages than that of its MPP.

Figure 3 shows the maximum electrical power density [Fig. 3(a)] and conversion efficiency [Fig. 3(b)] of nTiPV as a function of gap distance. Figure 4 rearranges the results from these figures to show conversion efficiency as a function of electrical power density. The results of the two kinds of "conventional" nTPV devices are also included: "ideal" nTPV assumes negligible ohmic losses and "real" nTPV assumes a PV cell with a series resistance of $10 \mathrm{~m} \Omega \cdot \mathrm{cm}^{2}$.

nTiPV generally outperforms nTPV, especially when considering a real nTPV device with non-negligible ohmic losses. Even in the case of an ideal nTPV (with negligible ohmic losses), nTiPV outperforms nTPV, provided that the inter-electrode distance is larger than $100 \mathrm{~nm}$ and the emitter workfunction is lower than $1.5 \mathrm{eV}$. The impact of the emitter workfunction is evident for small distances, where a low emitter workfunction (e.g., $\phi_{E}=1.3 \mathrm{eV}$ ) is needed to produce a high enough thermionic current and fully exploit the enhancement of the photovoltaic power generation in the near-field. In the case of larger emitter workfunctions, the low thermionic current limits the total current of the device and near-field effects are not fully exploited. At larger distances, the impact of the emitter's workfunction is negligible because the n'TiPV device is limited either by the photovoltaic current or by the space charge. Quite importantly, nTiPV produces a significantly higher power at larger (more feasible) gap distances [Fig. 3(a)]. For instance, an nTiPV device with an emitter (PV cell) surface workfunction of $1.3 \mathrm{eV}(1 \mathrm{eV})$ produces $6.73 \mathrm{~W} /$ $\mathrm{cm}^{2}$ for a gap distance of $100 \mathrm{~nm}$. This is 3.7 times more electrical power than an idealized nTPV device with negligible ohmic losses $\left(1.82 \mathrm{~W} / \mathrm{cm}^{2}\right)$
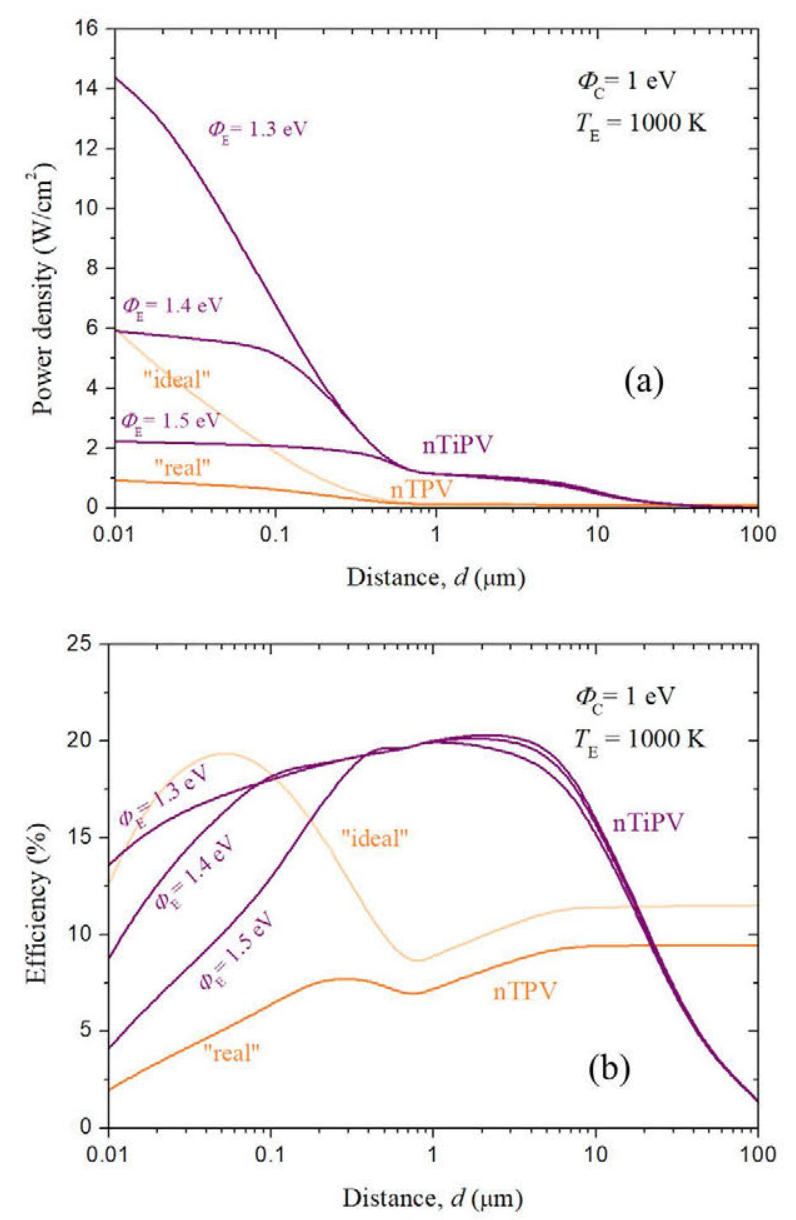

FIG. 3. Electrical power density (a) and conversion efficiency (b) of nTiPV and nTPV converters as a function of gap distance between the emitter and the PV cell. Different workfunctions of the emitter $\left(\phi_{\mathrm{E}}\right)$ are considered for $\mathrm{nTiPV}$. Ideal and real nTPV refer to the case with negligible ohmic losses and the more realistic case with a series resistance of $10 \mathrm{~m} \Omega \cdot \mathrm{cm}^{2}$, respectively. $R_{\text {lead }}$ is optimized at each distance to maximize the nTiPV conversion efficiency. The device area is $1 \mathrm{~cm}^{2}$.

and 10.7 times more electrical power than a realistic nTPV device having a series resistance of $10 \mathrm{~m} \Omega \cdot \mathrm{cm}^{2}\left(0.63 \mathrm{~W} / \mathrm{cm}^{2}\right)$. Besides, the conversion efficiency is similar to that of an idealized nTPV device ( $\sim 18 \%)$, but significantly higher than that of a realistic n'TPV device with non-negligible ohmic losses (6.4\%). Generally speaking, we can state that nTiPV operating at $1000 \mathrm{~K}$ theoretically enables reaching power densities and conversion efficiencies greater than $10 \mathrm{~W} / \mathrm{cm}^{2}$ and $15 \%$, respectively, while realistic n'PV is limited to $\sim 1 \mathrm{~W} / \mathrm{cm}^{2}$ and $\sim 7 \%$ (Fig. 4).

In summary, we have established a conceptual thermionicenhanced near-field thermophotovoltaic (nTiPV) device for the conversion of medium-grade heat into electricity. The converter comprises an InAs photovoltaic cell and a graphite emitter separated by a nanometric vacuum gap, both elements having engineered lowworkfunctions. Based on an analytical theoretical model that combines fluctuational electrodynamics and Langmuir theory, we have shown that n'TiPV produces significantly higher electrical power $\left(6.73 \mathrm{~W} / \mathrm{cm}^{2}\right)$ and conversion efficiency $(18 \%)$ than conventional near-field 


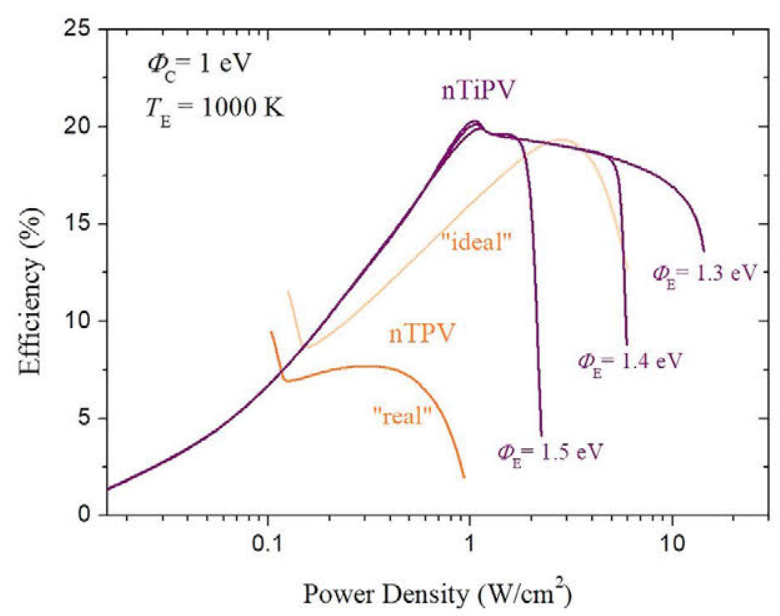

FIG. 4. Conversion efficiency as a function of electrical power density for nTiPV and nTPV converters, rearranged from the results shown in Fig. 3. Different workfunctions of the emitter $\left(\phi_{\mathrm{E}}\right)$ are considered for $\mathrm{nTiPV}$. Ideal and real nTPV refer to the case with negligible ohmic losses and the more realistic case with a series resistance of $10 \mathrm{~m} \Omega \cdot \mathrm{cm}^{2}$, respectively. $R_{\text {lead }}$ is optimized at each distance to maximize the nTiPV conversion efficiency. The device area is $1 \mathrm{~cm}^{2}$.

thermophotovoltaics (nTPV) using moderately large gap distances $(100 \mathrm{~nm})$. The major advantages are the elimination of the ohmic losses and the enhancement of the output voltage. According to these results, n'TiPV could significantly outperform current thermoelectric devices for the conversion of medium-grade heat sources into electricity.

This work was partially funded by the project AMADEUS, which has received funds from the European Union Horizon 2020 research and innovation program, FET-OPEN action, under Grant Agreement No. 737054. The sole responsibility for the content of this publication lies with the authors. It does not necessarily reflect the opinion of the European Union. Neither the REA nor the European Commission is responsible for any use that may be made of the information contained therein. A. Datas acknowledges postdoctoral fellowship support from the Spanish "Juan de la Cierva-Incorporación" program (IJCI-2015-23747). R. Vaillon is thankful to the Instituto de Energía Solar at the Universidad Politécnica de Madrid for hosting him and acknowledges the partial funding from the French National Research Agency (ANR) under Grant No. ANR-16CE05-0013. The authors acknowledge Daniele Trucchi and Alessandro Bellucci for the suggestion of using nanodiamond films as transparent low-workfunction coatings for the emitter and the PV cell.

\section{REFERENCES}

'D. B. Go, J. R. Haase, J. George, J. Mannhart, R. Wanke, A. Nojeh, and R. Nemanich, Front. Mech. Eng. 3, 13 (2017).

${ }^{2}$ K. A. A. Khalid, T. J. Leong, and K. Mohamed, IEEE Trans. Electron Devices 63, 2231 (2016).

${ }^{3}$ T. Bauer, Thermophotovoltaics: Basic Principles and Critical Aspects of System Design (Springer, 2011).

${ }^{4}$ D. L. Chubb, Fundamentals of Thermophotovoltaic Energy Conversion (Elsevier, 2007).

5. He and T. M. Tritt, Science 357, eaak9997 (2017).

${ }^{6}$ B. Wernsman, R. R. Siergiej, S. D. Link, R. G. Mahorter, M. N. Palmisiano, R. J. Wehrer, R. W. Schultz, G. P. Schmuck, R. L. Messham, S. Murray, C. S. Murray, F. Newman, D. Taylor, D. M. DePoy, and T. Rahmlow, IEEE Trans. Electron Devices 51, 512 (2004).
${ }^{7}$ D. N. Woolf, E. A. Kadlec, D. Bethke, A. D. Grine, J. J. Nogan, J. G. Cederberg, D. B. Burckel, T. S. Luk, E. A. Shaner, and J. M. Hensley, Optica 5, 213 (2018). ${ }^{8}$ R. He, D. Kraemer, J. Mao, L. Zeng, Q. Jie, Y. Lan, C. Li, J. Shuai, H. S. Kim, Y. Liu, D. Broido, C.-W. Chu, G. Chen, and Z. Ren, PNAS 113, 13576 (2016).

${ }^{9}$ D. L. Chubb, AIP Conf. Proc. 890, 297 (2007).

${ }^{10}$ N.-P. Harder and M. A. Green, Semicond. Sci. Technol. 18, S270 (2003).

"I. L. Pan, H. K. H. Choy, and C. G. Fonstad, IEEE Trans. Electron Devices 47, $241(2000)$.

${ }^{12}$ B. Zhao, P. Santhanam, K. Chen, S. Buddhiraju, and S. Fan, Nano Lett. 18, 5224 (2018).

${ }^{13}$ B. Song, A. Fiorino, E. Meyhofer, and P. Reddy, AIP Adv. 5, 053503 (2015).

${ }^{14}$ J. C. Cuevas and F. J. García-Vidal, ACS Photonics 5, 3896 (2018).

${ }^{15}$ E. Tervo, E. Bagherisereshki, and Z. Zhang, Front. Energy 12, 5 (2018).

${ }^{16}$ A. Fiorino, L. Zhu, D. Thompson, R. Mittapally, P. Reddy, and E. Meyhofer, Nat. Nanotechnol. 13, 806-8011 (2018).

${ }_{17}^{17}$ A. Datas, Appl. Phys. Lett. 108, 143503 (2016).

${ }^{18}$ F. A. M. Koeck and R. J. Nemanich, J. Appl. Phys. 112, 113707 (2012).

${ }^{19}$ F. A. M. Koeck, R. J. Nemanich, A. Lazea, and K. Haenen, Diamond Relat. Mater. 18, 789 (2009).

${ }^{20}$ A. Datas, A. B. Cristobal, C. del Cañizo, E. Antolín, M. Beaughon, N. Nikolopoulos, A. Nikolopoulos, M. Zeneli, N. Sobczak, W. Polkowski, M. Tangstad, J. Safarian, D. M. Trucchi, A. Bellucci, M. Girolami, R. Marx, D. Bestenlehner, S. Lang, A. Vitulano, G. Sabbatella, and A. Martí, in AIP Conference Proceedings (2018), p. 170004.

${ }^{21}$ A. Mezzi, P. Soltani, S. Kaciulis, A. Bellucci, M. Girolami, M. Mastellone, and D. M. Trucchi, Surf. Interface Anal. 50, 1-7 (2018).

${ }^{22}$ J. H. Lee, I. Bargatin, B. K. Vancil, T. O. Gwinn, R. Maboudian, N. A. Melosh, and R. T. Howe, J. Microelectromech. Syst. 23, 1182 (2014).

${ }^{23}$ R. Y. Belbachir, Z. An, and T. Ono, J. Micromech. Microeng. 24, 085009 (2014).

${ }^{24}$ K. Ito, K. Nishikawa, A. Miura, H. Toshiyoshi, and H. Iizuka, Nano Lett. 17, 4347 (2017).

${ }^{25}$ J. I. Watjen, B. Zhao, and Z. M. Zhang, Appl. Phys. Lett. 109, 203112 (2016).

${ }^{26}$ M. Ghashami, H. Geng, T. Kim, N. Iacopino, S. K. Cho, and K. Park, Phys. Rev. Lett. 120, 175901 (2018).

${ }^{27}$ K. Ito, A. Miura, H. Iizuka, and H. Toshiyoshi, Appl. Phys. Lett. 106, 083504 (2015).

28. DeSutter, Thermophotovoltaic Energy Conversion: Emitter Optimization, Near-Field Radiative Recombination and Near-Field Radiative Devices (University of Utah, 2019).

${ }^{29}$ G. N. Hatsopoulos and E. P. Gyftopoulos, Thermionic Energy Conversion (The MIT Press, Cambridge, Massachusetts, 1979).

${ }^{30}$ S. M. Rytov, Y. A. Kravtsov, and V. I. Tatarskii, Principles of Statistical Radiophysics 3: Elements of Random Fields (Springer-Verlag, Berlin Heidelberg, 1989).

${ }^{31}$ M. Francoeur, M. Pinar Mengüiç, and R. Vaillon, J. Quant. Spectrosc. Radiat. Transfer 110, 2002 (2009).

${ }^{32}$ M. P. Bernardi, O. Dupré, E. Blandre, P.-O. Chapuis, R. Vaillon, and M. Francoeur, Sci. Rep. 5, 11626 (2015).

${ }^{33}$ E. Blandre, P.-O. Chapuis, and R. Vaillon, Sci. Rep. 7, 15860 (2017).

${ }^{34}$ M. E. Levinshtein, Handbook Series on Semiconductor Parameters, Vol. $1 \mathrm{Si}$, Ge, C (Diamond), GaAs, GaP, GaSb, InAs, InP, InSb (World Scientific, 1996).

${ }^{35}$ M. Sotoodeh, A. H. Khalid, and A. A. Rezazadeh, J. Appl. Phys. 87, 2890 (2000).

${ }^{36}$ T. Teraji, S. Koizumi, and H. Kanda, Appl. Phys. Lett. 76, 1303 (2000).

${ }^{37}$ J. E. Gerbi, O. Auciello, J. Birrell, D. M. Gruen, B. W. Alphenaar, and J. A. Carlisle, Appl. Phys. Lett. 83, 2001 (2003).

${ }^{38}$ W. W. Anderson, Infrared Phys. 20, 363 (1980).

${ }^{39}$ R. W. Gammon and E. D. Palik, J. Opt. Soc. Am., JOSA 64, 350 (1974).

${ }^{40} \mathrm{~S}$. Adachi, Optical Constants of Crystalline and Amorphous Semiconductors: Numerical Data and Graphical Information (Springer US, 1999).

${ }^{41}$ I. Latella, P. Ben-Abdallah, S.-A. Biehs, M. Antezza, and R. Messina, Phys. Rev. B 95, 205404 (2017).

${ }^{42}$ A. M. Zaitsev, Optical Properties of Diamond: A Data Handbook (SpringerVerlag, Berlin Heidelberg, 2001).

${ }^{43}$ J. Lagarias, J. Reeds, M. Wright, and P. Wright, SIAM J. Optim. 9, 112 (1998). 